NASA Technical Memorandum 83714

\title{
Some Fundamental Aspects of Solidification in a Supercooled Melt
}

V. Laxmanan

Lewis Research Center

Cleveland, Ohio

Prepared for the

Fifth Conference on Rapid Quenching and Solidification of Metals (RQ5)

Wurzburg, West Germany, September 3-7, 1984

\section{NASA}


SOME FUNDAMENTAL ASPECTS OF SOLIDFICATION IN A SUPERCOOLED MELT

V. Laxmanan*

National Aeronautics and Space Administration

Lewis Research Center

Cleveland, Ohio 44135

\section{INTRODUCTION}

Dendritic growth in a pure melt can only occur in the presence of a negative temperature gradient in the bulk liquid ahead of the dendrite tips, in other words, in a supercooled bath since even a small positive temperature gradient in the liquid can suppress dendrite formation (1). In an alloy melt, however, dendritic growth occurs quite readily with both positive and negative temperature gradients in the bulk liquid. This is because of the "constitutional supercooling" developing in the bulk liquid due to the compositional changes accompanying the growth process $(2-4)$. The main purpose of this paper is to discuss dendritic growth as it occurs in an "undercooled" or "supercooled" melt.

\section{DENDRITE TIP UNDERCOOLING VERSUS BATH} SUPERCOOLING

When solidification occurs in a positive temperature gradient, in an alloy melt, the temperature of the advancing solid-liquid interface (be it planar, cellular or dendritic) is always depressed below the equilibrium liquidus temperature, $T_{L}$, for the initial alloy composition, $C_{0}$, by an amount $\Delta T$ which includes : the contributions due to the compositional changes occurring in the liquid, curvature, and kinetic effects $(4)$. During cellular or dendritic growth, this depression in the interface temperature is usually referred to as the "tip undercooling" $(5,6)$. It is important to recognize that steady-state cellular or dendritic growth will always occur with such an "undercooling", even if the first solid were to nucleate at exactly the liquidus temperature $T_{L}$. Likewise, if nucleation occurs after the molten alloy bath has been cooled to some temperature, $T_{\infty}$, well below the liquidus, the temperature at the advancing interface will quickly rise to some value above $T_{\infty}$. The temperature gradient, $G_{L}$, in the bulk liquid ahead of the advancing tips will, therefore, become negative as shown schematically in Figure 1 . The temperature gradient within the interdendritic regions, $G_{j}$, may, however, be positive or negative ${ }^{(7)}$. Here it will be assumed that $G_{i}>0$. Note that the dendrite tip temperature, $T_{t}$, is still depressed below $T_{L}$ by an amount $\Delta T$ given by:

\footnotetext{
*Case Western Reserve University, Cleveland, Ohio, and NASA Resident Research Associate.
} 


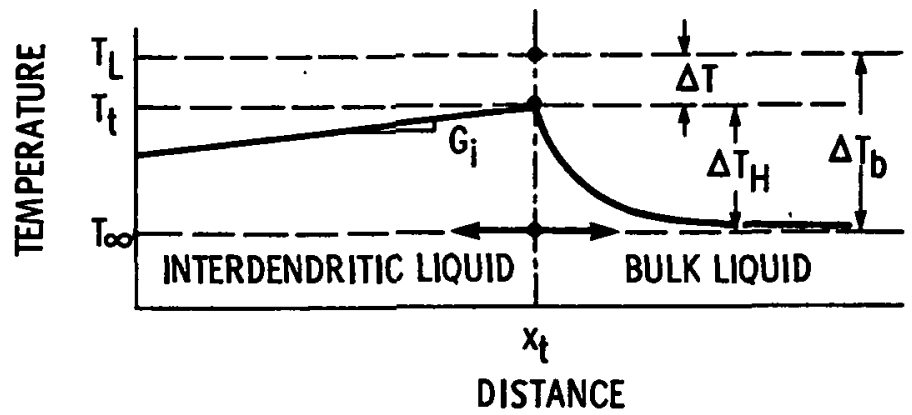

Figure 1: Temperature distribution during dendritic growth in a supercooled alloy melt.

$$
\frac{\Delta T}{\Delta T_{0}}=\left\{\frac{a k}{k-T}+\frac{R k}{D_{L}}\left(1-\frac{a k}{k-T}\right) \lambda_{c} r_{t}+\frac{2 e_{c} k}{r_{t}}\right\}
$$

Here $\lambda_{c}$ is a dimensionless quantity related to the "effective" diffusion distance $\delta$ in the bulk liquid ahead of the dendrite tips; $\delta=\lambda_{c} r_{t}$, $r_{t}$ being the tip radius. The other symbols in equation have been defined in a companion paper in this volume ${ }^{(4)}$. Equation (1) above assumes that the kinetic undercooling, $\Delta \mathrm{T}_{k} \simeq 0$ and that the Peclet number $p \ll 1 / 2 \lambda_{c}(1-k) ; p=R r_{t} / 2 D_{L}$. The bulk liquid far away from the dendrite tip is depressed, below $T_{L}$, by an amount $\Delta T_{b}=$ $\left(T_{L}-T_{\infty}\right)$. In what follows, $\Delta T_{b}$ will be referred to as the bath "supercooling". The term "undercooling" will be used exclusively to describe the depression in the tip temperature. Finally, it must be emphasized here that the dimensionless parameter $a=D_{L} G_{j} / m_{L} R C_{0}$ includes only the interdendritic temperature gradient $G_{i}(2,3)$.

\section{DENDRITE STRUCTURE IN A SUPERCOOLED MELT}

Recent direct, in-situ, observations on dendrites growing in transparent materials such as pure succinonitrile ( $\mathrm{SCN})$, pivalic acid and in dilute alloys of SCN containing argon or acetone $(8,9)$ indicate that the dendrite tip is a paraboloid of revolution with side-branches developing at a short distance behind the tip. However, this characteristic branched structure begins to degenerate to a simpler cylindrical form at moderately large bath supercoolings, whereas at very large bath supercoolings (typically about $170 \mathrm{~K}$ in most ferrous alloys) there is an abrupt transition from a cylindrical to a spherical microstructure ${ }^{(10)}$.
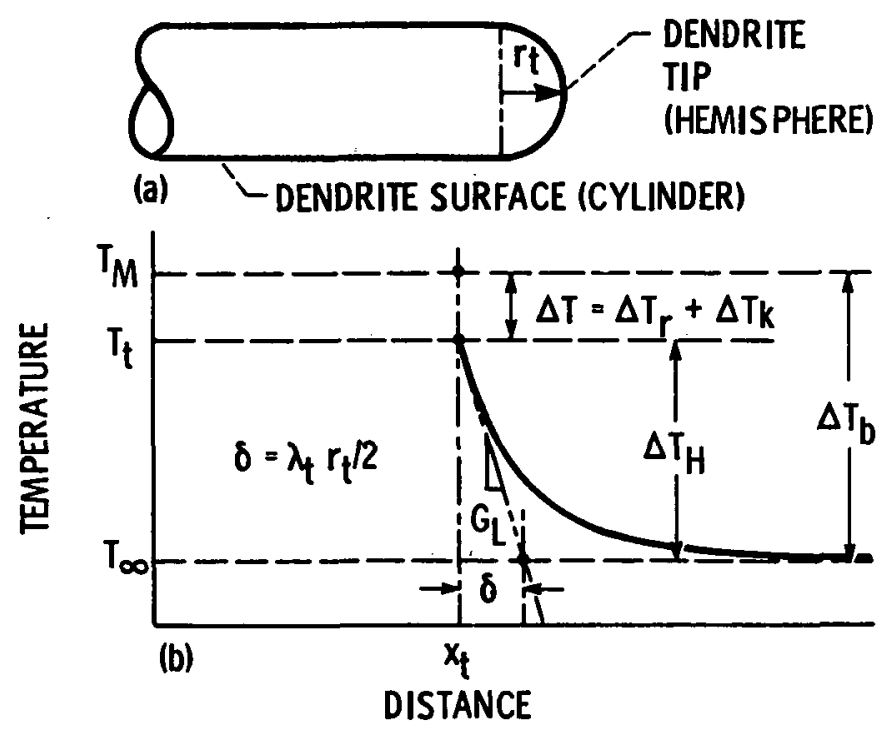

Figure 2: Dendritic growth in a supercooled pure melt.

The so-called "Fisher dendrite" which is assumed to have a hemispherical tip and a cylindrical body ${ }^{(11)}$, Figure $2 a$, has a morphology remarkably similar to the microstructure observed in moderately supercooled melts. The solution to this dendrite shape, as it applies to a supercooled pure melt will be discussed next followed by a discussion of solidification in a supercooled alloy.

4. DENDRITIC GROWTH IN A SUPERCOOLED PURE MELT

Figure 2b illustrates schematically the temperature distribution in the bulk liquid. Let $G_{L}$ be the temperature gradient in the liquid at exactly the dendrite tip. $G_{L}=-\left(T_{t}-T_{\infty}\right) / \delta$ where $\delta$ is now the "effective" thermal diffusion distance. This is simply the distance into the liquid at which the temperature would drop to $T_{\infty}$ if the gradient in the liquid had a : constant value $G_{L}$. The tip temperature $T_{t}$ is depressed below the equilibrium melting point $T_{M}$ by an amount $\Delta T=\left(T_{M}-T_{t}\right)=\Delta T_{r}+\Delta T_{k}$ where $\Delta T_{r}$ and $\Delta T_{k}$ are respectively the depression in tip temperature (or "undercooling") 
attributed to the Gibbs-Thomson effect and the kinetic effect $(12)$ associated with the growth velocity, $R$, of the tip. "Here it will be assumed that $\Delta T_{k} \simeq 0$. Thus, it may be argued that only a portion, $\Delta T$, of the total bath supercooling, $\Delta T_{b}$, is required to ensure that the dendrite tip is in "equilibrium" with the bath. The remainder, $\Delta T_{H}$, is required to dissipate the heat of fusion generated at the tip. A simple heat balance at the tip gives:

$$
T_{t}=T_{\infty}+\frac{L}{C_{p}}\left(\frac{\lambda_{t} R r_{t}}{2 \alpha_{L}}\right)
$$

where $L$ is the heat of fusion per unit volume, $C_{p}$ is the volumetric specific heat of the liquid, $\alpha_{L}$ is the thermal diffusivity of the liquid and $\delta=\lambda_{t} r_{t} / 2$.

For equilibrium, the tip temperature must al so be given by:

$$
T_{t}=T_{M}-\frac{L}{C_{p}}\left(\frac{2 d_{0}}{r_{t}}\right)
$$

where $d_{0}$ is a characteristic length called the capillary length ${ }^{(8)}$. Combining equations (2) and (3) above yields

$$
\Delta \theta=\frac{T_{M}-T_{\infty}}{L / C_{p}}=\lambda_{t}\left(\frac{R r_{t}}{2 \alpha_{L}}\right)+\frac{2 d_{0}}{r_{t}}
$$

Figure 3 plots schematically the tip temperatures given by equations (2) and (3) respectively as a function of the tip radius. Clearly, steady-state solidification can only occur when the tip radius has the value $r_{1}$ or $r_{2}$ given by the intersection of these two plots. These radii are given by the following quadratic expression for $r_{t}$ :

$$
r_{t}^{2}\left(R / R_{m}\right)-4 R_{c} r_{t}+4 r_{c}^{2}=0
$$

where $r_{c}=2 d, \Delta \theta$ is the critical nucleation radius $\left(\mathcal{T}_{3}, 14\right)^{0}$ and $R_{m}=\alpha_{L} d_{0} / \lambda_{t} r_{c}^{2}$. For $R>R_{m}$, the roots of the above quadratic equation become imaginary. $R_{m}$ is thus the maximum

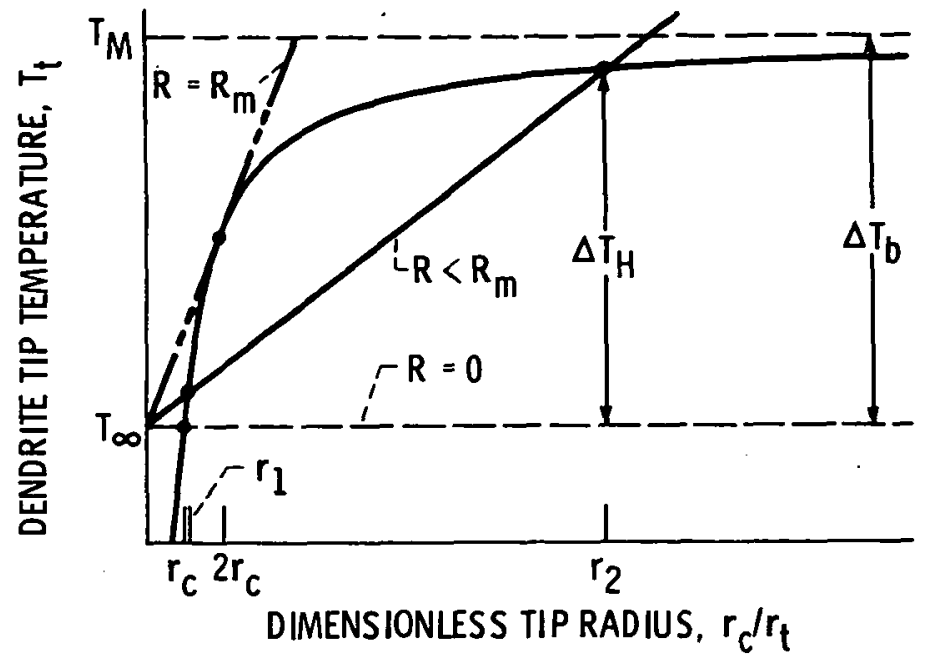

Figure 3: Schematic plot of tip temperature versus tip radius given by equations (2) and (3).

possible growth rate, for a given $\Delta T_{b}$. Previous analyses have simply assumed that the dendrite grows at this maximum growth rate $(8,12)$, for which the tip radius $r_{t}=2 r_{c}$ and the tip temperature $T_{t}=\left(T_{M}+T_{\infty}\right) / 2$. Thus, for $R=R_{m}, \Delta T=\Delta T_{H}$; so that exactly one-half of the total bath supercooling $\Delta T_{b}$ is required to dissipate the heat of fusion.

Figure 3 clearly illustrates that the "maximum velocity" hypothesis has no firm basis and that steady-state growth can occur at a growth rate $R<R_{m}$. However, it is not immediately obvious what dictates the choice between the two possible radii $r_{1}$ and $r_{2}$. Rewriting equation (5) as follows:

$$
\sigma=\frac{2 \alpha_{L} d_{0}}{R r_{t}^{2}}=\frac{\lambda_{t}}{2}\left(\frac{r_{t}}{r_{c}}-1\right)^{-1}
$$

Here $\sigma$ is a dimensionless parameter characteristic of the dendritic growth process (14). For $R \ll R_{m}$, this parameter, for the larger radius $r_{2}$ given by equation (5) is approximately equal to 


$$
\sigma \simeq \frac{\lambda}{8}\left(\frac{R / R_{m}}{1-R / 4 R_{m}}\right)
$$

and, for the smaller radius $r_{1}, \sigma \simeq-\lambda_{t} / 2$. For $R=R_{m}, \sigma$ is exactly $\lambda_{t} t^{\prime 2}$.

It is important to note here that the parameter $\sigma$ obtained here is exactly the "tip stability" parameter obtained by Langer and Muller-Krumbhaar (14). o follows naturally from the analysis of steady-state solidification as discussed here. It may be calculated simply by knowing either the dendrite tip growth rate, $R$, or the tip radius, $r_{t}$, provided $\lambda_{t}$ is known. It has usually been assumed that $\lambda_{t} \simeq 1$ but this need not be true $(3,4)$. Thus, for pure $S C N(15)$, the measured tip radius at $\Delta \theta=0.001$ is about $30 \mu \mathrm{m}$ and the observed growth rate $R=10 \mu \mathrm{m} / \mathrm{sec}$. At this supercooling, $r_{c}=$ $0.54 \mu \mathrm{m}$ and $R_{m}=1037 / \lambda_{t} \mathrm{~m} / \mathrm{sec}$, giving $\lambda_{t} \simeq 7.466$. Thus, the "effective" thermal diffusion distance is almost four times the tip radius at this supercooling.

Langer and Muller-Krumbhaar observed that for $\sigma$ greater than a critical value, $\sigma^{*}$, sidebranching along the dendrite surface greatly decreases. Thus, it may be argued that "cylindrical" (and also, "spherical") growth morphology observed in moderately supercooled melts correspond to dendritic growth with large values of $\sigma$. This simply implies that dendritic growth will occur at smaller values of the ratio $r_{t} / r_{c}$ with increasing bath supercoolings.

\section{DENDRITIC GROWTH IN A SUPERCOOLED} ALLOY MELT

In an alloy melt, steady-state solidification will occur with a tip radius which satisfies both equations (1) and (2) simultaneously:

$\frac{\Delta T_{b}-a m_{L} C_{0}}{\Delta T_{0}}=2 \lambda_{c} \beta k(1+\xi) \frac{R r_{t}}{2 D_{L}}+\frac{2 e_{c} k}{r_{t}}$ where $\xi=\frac{D_{L} \lambda_{t} / 2^{\prime}}{\lambda_{c} \beta k \alpha_{L} \Delta T_{0}\left(C_{p} / L\right)}$

and $\beta=1-a k /(k-1)$. In metal alloys, $D_{L} / \alpha_{L}$ is typically about $10^{-3}$, so that, $\xi$ is negl igibly small, especially at large growth rates for which $\beta \simeq 1$ since $a \simeq 0$. Thus equations (4) and (8) are remarkably similar if $a \simeq 0$ and $\xi \simeq 0$ with $\lambda_{t}, \alpha_{L}$ and $d_{0}$ being replaced by their chemical counterparts $\lambda_{c}$, $D_{L}$ and $\ell_{c}$. For a pure metal the partition ratio $k=1$. Note that $a \simeq 0$ both for very large growth rates and also if the interdendritic concentration gradient, $G_{i} / m_{L}$, is negligibly sma $17^{(3)}$. Thus it may be argued that $\mathrm{a} \simeq 0$ when spherical particles grow in a supercooled alloy melt, since within a spherical particle there are no "interdendritic channels" through which solute diffusion takes place. Equation (8) may, therefore, al so be used to describe solidification in a highly supercooled melt.

Rewriting equation (8) yields a dimensionless parameter $\sigma_{c}=2 l_{c} D_{L} / R r_{t}^{2}$, given by:

$$
\sigma_{c}=\lambda_{c}(1+\xi)\left(\frac{r^{*}}{r_{t}}-1\right)
$$

where $r^{*}=\frac{D_{L} / R k}{\lambda_{c}\left(1+\xi_{j}\right) \Delta T_{0} / \Delta T_{b}}$

Note that $\sigma_{C}$ is the chemical counterpart of the "tip stability" parameter $\sigma$ defined earlier ${ }^{(16)}$. For $r_{t}>r^{*}, \sigma_{c}$ will become negative. $r^{*}$ thus represents the maximum particle size or the tip radius for a given growth rate and bath supercooling. The smallest particle size is, of course, the critical nucleation radius, $r_{c}$, which may be obtained from equation ( 8 ) directly by setting $R=0$, with $\beta=1, a=0$. Thus, $r_{c}=2 l_{c} k \Delta T_{0} / \Delta T_{b}$, and, 


$$
\frac{r^{*}}{r_{c}}=\frac{D_{L} / 2 e_{c} k^{2}}{R}\left(\frac{\Delta T_{b}}{\Delta T_{0}}\right)^{2} \frac{1}{\lambda_{c}(1+\xi)}
$$

Recall that $R_{a}=D_{L} / \ell_{c} k^{2}$ is the growth velocity above which a planar interface will remain stable according to the "absolute stability" criterion $(4,17)$. At these large growth rates $\sigma_{c}$ becomes negligibly small since $\lambda_{c}$ also tends to zero $(3,4)$ at large values of $R$. This may be interpreted as implying "solute trapping" or "partitionless" solidification for which $k$ tends to unity. More importantly, equation (10) clearly indicates that the "tip stability" parameter $\sigma_{c}$ is the underlying fundamental quantity governing these transitions. Finally, it may be noted that the stability parameters $\sigma$ and $\sigma_{c}$ are both related to the respective dimensionless length scales $\lambda_{t}$ and $\lambda_{c}$.

\section{REFERENCES}

1. F. Weinberg and B. Chalmers, Can. J. Phy. 29 (1951) 382.

2. V. Laxmanan, "Constitutional Supercooling at the Dendrite Tip", submitted to Met. Trans. A.

3. V. Laxmanan, "Dendritic Solidification at Large Growth Rates", submitted to Met. Trans. A.

4. V. Laxmanan, "Interface Morphology During Rapid Solidification" these proceedings.

5. M.H. Burden and J.D. Hunt, J. Cryst. Growth, 22 (1974) 109.

6. M.C. Flemings, in Met. Treatises, Eds. J.H. Tien and J.F. Elliott, ASM (1981) p.

7. V. Laxmanan, "A Model for Dendritic Growth in a Supercooled Alloy Melt", to be published.

8. S.C. Huang and M.E. Glicksman, Acta Met., 29 (1981) 701.

9. M.E. Glicksman, private communication.

10. T.Z. Kattamis and M.C. Flemings, Trans. AIME, 236 (1966) 1523.

11. B. Chalmers, Principles of Solidification, John Wiley and Sons, New York, (1964) 105.

12. R. Trivedi, H. Franke and R. Lacmann, J. Cryst. Growth, 47 (1979) 389.

13. S.R. Coriell and D. Turnbull, Acta Met, 30 (1982) 2135.

14. J.S. Langer, Rev. Mod. Phys., 52 (1980) 1.

15. M.E. Glicksman, R.J. Schaeffer and J.D. Ayers, Met. Trans. A, 7A (1976) 1747.

16. V. Laxmanan, "Dendritic Solidification in a Binary Alloy Under an Imposed Thermal Gradient: Minimum Undercooling versus Tip Stability Criterion", submitted to Met. Trans. B.

17. W.W. Mullins and R.F. Sekerka, J. Appl. Phys., 35 (1964) 444. 


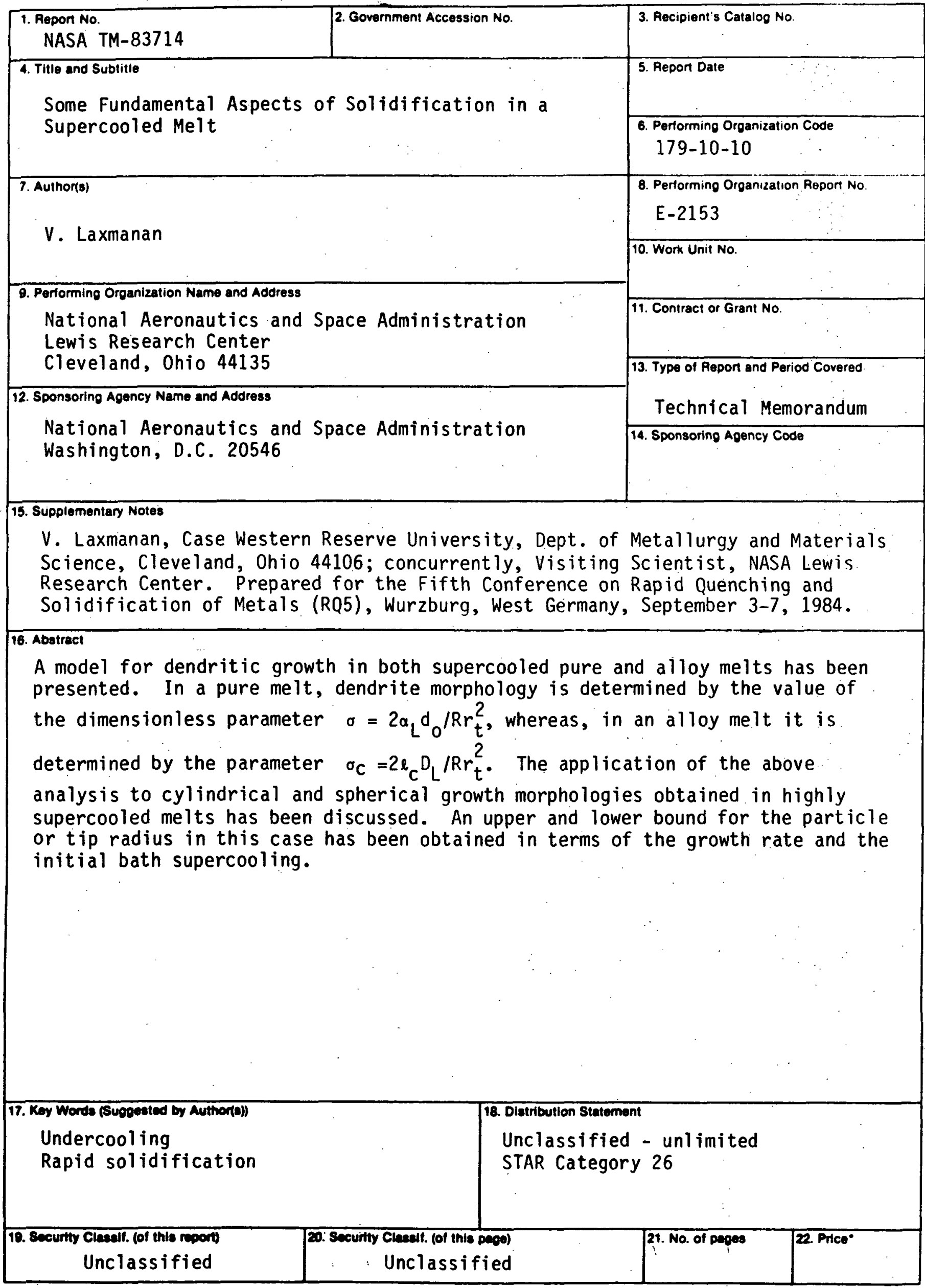


National Aeronautics and

Space Administration

Washington, D.C.

20546

Official Business

Penalty for Private Use, $\$ 300$
SPECIAL FOURTH CLASS MAIL BOOK

Portege and Fees Paid

National Aeronautics and

Sowce Administration

NASA 451 